\title{
Enfermería y Antropología en Brasil: Relaciones, Dilemas y Desafíos
}

\section{Nursing and Anthropology in Brazil: Relations, Dilemmas and Challenges}

\section{Enfermagem e Antropologia no Brasil: Relações, Dilemas e Desafios}

\author{
Lucas Pereira de Melo
}

${ }^{1}$ Doutorando do Programa Interunidades de Doutoramento em Enfermagem dos campi São Paulo e Ribeirão Preto da Universidade de São Paulo. Professor da Faculdade de Enfermagem, Pontifícia Universidade Católica de Campinas, São Paulo, Brasil.

Cómo citar este artículo en edición digital: Pereira de Melo, L. (2013) Enfermagem e Antropologia no Brasil: Relações, Dilemas e Desafios. Cultura de los Cuidados (Edición digital) 17, 36. Disponible en: http://dx.doi.org/10.7184/cuid.2013.36.08

Correspondencia: Lucas Melo. Rua Teresa Bonatto Signori, 51, Jd. Santa Genebra II. Campinas-SP-Brasil. CEP 13084777.

Correo electrónico: lucasenf@yahoo.com.br

Recibido: 10/04/2013. Aprobado: 22/06/2013

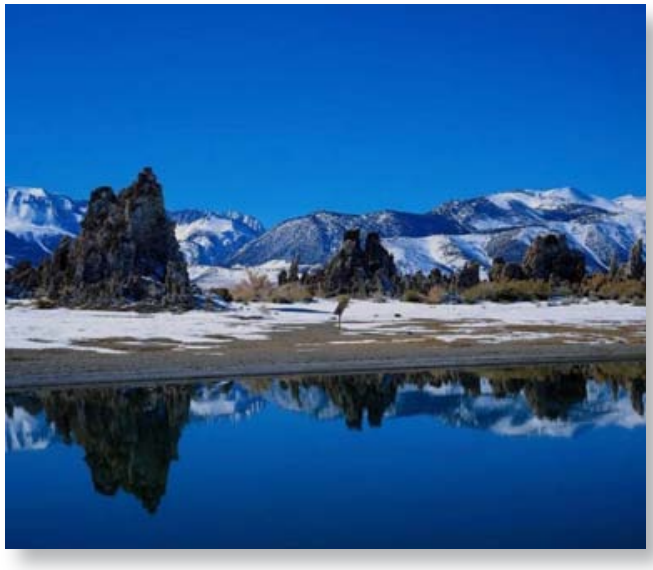

\section{ABSTRACT}

Taking as a starting point to finding the invisibility of nursing scientific production in anthropological perspective on the literature reviews on anthropology of health in Brazil, the aim of this essay is to make a brief critical discussion of nursing and anthropology punctuating the particularities of the Brazilian context compared with countries like United States, England and Spain, and especially discuss the dilemmas (problems and limits) in the relations existing between the two disciplines and indicate some challenges to its development and consolidation. Finally, the enrollment of the arguments and comparative analysis of building this relationship in different countries allowed viewing certain hypotheses that aim to contribute to future discussions and studies in Brazil.

Keywords: Nursing. Anthropology. Specialities, Nursing. Transcultural Nursing. Philosophy, Nursing.

\section{RESUMEN}

Tomando como punto de partida la invisibilidad de la producción científica de enfermería, en la perspectiva antropológica, en las revisiones de la literatura sobre antropología de la salud en Brasil, el objetivo de este artículo es realizar un breve análisis crítico de la relación entre enfermería y antropología puntuando las particularidades del contexto brasileño en comparación con países como Estados Unidos, Gran Bretaña y España y, en especial, discutir los dilemas (problemas y límites) en las relaciones existentes entre las dos disciplinas y señalar algunos desafíos para su desarrollo y consolidación. Por último, los argumentos y análisis comparativo de la construcción de esta relación en los diferentes países permiten ver 
ciertas hipótesis que apuntan a contribuir a los futuros debates y estudios en Brasil.

Palabras clave: Enfermería. Antropología. Especialidades de Enfermería. Enfermería Transcultural. Filosofía en Enfermería.

\section{RESUMO}

Tendo como ponto de partida a constatação da invisibilidade da produção científica da enfermagem na perspectiva antropológica nas revisões da literatura sobre antropologia da saúde no Brasil, o objetivo deste ensaio crítico é fazer uma breve discussão sobre enfermagem e antropologia pontuando as particularidades do contexto brasileiro em comparação com países como Estados Unidos, Inglaterra e Espanha e, principalmente, problematizar os dilemas (problemas e limites) existentes nas relações entre as duas disciplinas e indicar alguns desafios para seu desenvolvimento e consolidação. Por fim, o arrolamento dos argumentos e a análise comparativa da construção dessa relação em diferentes países permitiram visualizar algumas hipóteses que visam contribuir para estudos e discussões futuras no Brasil.

Palavras-chave: Enfermagem. Antropologia. Especialidades de Enfermagem. Enfermagem Transcultural. Filosofia em Enfermagem.

\section{INTRODUÇÃO}

O estudo das relações entre antropologia, saúde e doença, bem como das instituições de saúde e seus agentes de cura é um campo já consolidado, tanto no Brasil quanto em outros países, desde meados do século XX. No Brasil, há uma extensa produção acadêmica que aborda as contribuições da antropologia para as disciplinas da área de saúde, a institucionalização da chamada Antropologia da Saúde, revisões das pesquisas desenvolvidas nesse campo e suas questões e desafios atuais. Porém, há uma incipiente produção sobre as relações entre enfermagem e antropologia.

Durante minha formação de pósgraduação na Universidade Estadual de Campinas (mestrado) e na Universidade de São Paulo (doutorado), fui orientado e tive contato direto, por meio de cursos, seminários e encontros informais, com alguns dos precursores da Antropologia da Saúde brasileira e, consequentemente, com parte considerável da literatura antropológica sobre saúde, doença e cura. Por estar inserido em Programas de Pós-graduação em Enfermagem, transitei, paralelamente, dentro das interfaces que relacionam a enfermagem e a antropologia, sobretudo com as pesquisas em enfermagem com referencial antropológico. Com o olhar atento ao desenvolvimento desses conjuntos de produções (da antropologia da saúde e da enfermagem na perspectiva antropológica), pude constatar uma invisibilidade da produção científica da enfermagem nas revisões da literatura sobre antropologia da saúde no Brasil, realizadas por pesquisadores vinculados aos Departamentos de Antropologia ou de Saúde Coletiva/Saúde Pública.

Diante disso, passei a investigar as relações entre essas disciplinas, bem como suas contribuições e influências. Além disso, serviu de contraponto nessas análises a participação em eventos e/ou como associado na Transcultural Nursing Society (Estados Unidos), na Asociación de Historia y Antropología de los Cuidados (Espanha), na Medical Anthropology Young Scholars (MAYS) uma rede de pesquisadores da European Association of Social Anthropologists e na Associação Brasileira de Antropologia (ABA).

Nesse sentido, o objetivo deste ensaio crítico é fazer uma breve discussão sobre enfermagem e antropologia pontuando as particularidades 
do contexto brasileiro em comparação com países como Estados Unidos, Inglaterra e Espanha e, principalmente, problematizar os dilemas (problemas e limites) existentes nas relações entre as duas disciplinas e indicar alguns desafios para seu desenvolvimento e consolidação. Parte-se do pressuposto que para inverter essa economia de invisibilidade faz-se necessária uma reflexão e uma análise do que se tem produzido até o momento; processo este que deve incluir os pesquisadores da enfermagem, da antropologia em geral e da antropologia da saúde em particular.

\section{RELAÇÕES ENTRE ENFERMAGEM E ANTROPOLOGIA: OS CASOS AMERICA- NO, INGLÊS E ESPANHOL}

As relações entre enfermagem e antropologia têm sido discutidas em diversas publicações nacionais e internacionais (Noble, 1964; Leininger, 1970; 2001; Dougherty \& Tripp-Reimer, 1985; Littlewood, 1989; Mulhall, 1996; Cartana \& Heck, 1997; Martorell Poveda, 2001; Siles et al., 2001). A enfermagem norteamericana pode ser considerada o berço das relações entre as disciplinas de enfermagem e antropologia. Nos Estados Unidos (EUA), a II ${ }^{\mathrm{a}}$ Guerra Mundial foi um marco na construção das relações entre as duas disciplinas (Dougherty \& Tripp-Reimer, 1985). Antes da II a Guerra Mundial já havia alguns trabalhos de enfermeiras de saúde pública sobre as dimensões culturais implicadas no cuidado publicados na Public Health Nursing Quarterly. No entanto, apenas depois da II ${ }^{a}$ Guerra Mundial, devido a experiência das enfermeiras que serviram nos campos de batalha, a ênfase nas diferenças culturais entre grupos étnicos puderam ser mais bem percebidas e, posteriormente, ensinadas em cursos de Enfermagem.
Cumpre destacar que, desde 1937, a National League of Nursing havia tornado obrigatório o oferecimento de pelo menos 10 horas-aula de Ciências Sociais, por semestre, nos cursos de graduação em Enfermagem. Nesse processo, a antropóloga Esther Lucille Brown teve uma importância particular (Dougherty \& Tripp-Reimer, 1985).

Nos anos 1950 o projeto da Russell Sage Foundation realizado na Cornell University School of Nursing entre os anos 1954 e 1957 convidaram vários professores renomados para oferecer cursos de Antropologia e Sociologia naquela universidade. Dentre eles destacamse: Margaret Mead, Renee Fox, Rhoda Metraux e August Hollingshead. Já nos anos 1960, com o Nurse Scientists Program, de abrangência nacional, aumentou-se o incentivo para que enfermeiros obtivessem o grau de Doutor. Nesse contexto, alguns enfermeiros cursaram Doutorado em Antropologia, sendo a Dra. Madeleine Leininger (1925-2012) a primeira enfermeira profissional do mundo a obter o título de Doutora em Antropologia, em 1965, pela University of Washington (Dougherty \& Tripp-Reimer, 1985).

O pioneirismo da Dra. Leininger na consolidação da relação entre Enfermagem e Antropologia deve-se, principalmente, à criação de um subcampo dentro da Enfermagem: a Enfermagem Transcultural. Herdeira de uma tradição própria do Culturalismo Americano (ela recebeu grande influência de Ruth Benedict e Margaret Mead), Dra. Leininger dedicou-se ao estudo das diferenças e similaridades culturais entre grupos étnicos que vivem nos EUA e em outros países, como forma de desenvolver o que ela chamou de "cuidado culturalmente congruente". Para a identificação desses traços culturais, a autora estabeleceu um método de 
pesquisa qualitativo específico para este fim, fortemente fundamentado na Etnografia: a Etnoenfermagem.

Em 1968, Dra. Leininger fundou e foi a primeira presidente do Committee on Nursing and Anthropology (CONAA) ligado à Society for Medical Anthropology órgão da American Anthropological Association. Nos anos 1970, Leininger sentiu-se descontente com a perspectiva da Antropologia Médica que vigorava no CONAA: os antropólogos e enfermeiros no CONAA estavam interessados em doenças específicas (sintomas e tratamentos) e no modelo médico, sem levar em consideração as bases teóricas do conhecimento da enfermagem sobre o cuidado e a saúde (Leininger, 2001). Nessa época a Enfermagem Transcultural já era uma área de estudo e prática clínica academicamente reconhecida, com cursos e programas oferecidos nos EUA e em outros países. Essas divergências fizeram com que a Dra. Leininger fundasse e presidisse, em 1974, a Transcultural Nursing Society e o Journal of Transcultural Nursing do qual ela foi a primeira editora.

Destaca-se ainda a criação, em 1980, da American Nurses' Association's Council on Inter-Cultural Diversity. Além disso, é notório o papel dessas organizações na introdução de disciplinas de Antropologia e Enfermagem Transcultural nos currículos dos cursos de Enfermagem americanos (Dougherty \& TrippReimer, 1985).

Diferente da enfermagem norteamericana, o desenvolvimento de relações entre enfermagem e antropologia na Inglaterra se dá por outra via. A enfermagem britânica recebeu influências da Antropologia Social. A tradição da Antropologia Social britânica inaugurada por Malinowsky e Radcliffe-Brown introduziu uma perspectiva de totalidade do fato psicológico e social. Nessa perspectiva, “o fato social só tem sentido no interior do contexto cultural que lhe conjuntura" (Queiroz, 2003). Posteriormente, autores como Gluckman, Leach e Turner inserem uma abertura teórica para incluir o indivíduo e a dimensão cotidiana do fato social e psicológico, em contraposição à postura funcionalista de Malinowsky, incluindo a noção de conflito e agência individual.

Dessa forma, o campo de estudo da antropologia social são os comportamentos e relações sociais dentro de um dado contexto como a família, o sistema de parentesco, as situações de trabalho, as organizações políticas, os grupos religiosos. Dada sua orientação funcionalista, o antropólogo social estuda as instituições sociais como partes interdependentes de um sistema social total e acredita que o estudo de uma situação social qualquer fora do seu contexto social pode distorcer o significado da situação estudada (Noble, 1964).

As enfermeiras britânicas produziram críticas contundentes à ênfase dada pela Enfermagem Transcultural às minorias étnicas (imigrantes, refugiados, estrangeiros em geral). As inglesas criticam as americanas nos seguintes pontos: o entendimento de cultura como significado de minorias étnicas e um fenômeno reificado e isolado; o uso de questionários, de tipo checklists, para "catalogar" as práticas de cuidado de pessoas de culturas diferentes; a dificuldade em analisar as relações de saber-poder existentes entre enfermeiro-paciente durante a produção do cuidado; a consideração superficial das forças sociopolíticas presentes nas interações sociais, principalmente nos encontros clínicos; e o desenvolvimento de análises que pouco 
desafia a dominância do sistema político e cultural do Ocidente (Mulhall, 1996).

Em contrapartida, na enfermagem britânica, vê-se a produção de modelos teóricos e empíricos que incorporam os saberes produzidos pela antropologia médica no Processo de Enfermagem, valorizando a compreensão dos fenômenos de saúde, doença e cuidado dentro do sistema simbólico do indivíduo adoecido (Littlewood, 1989). Nesse sentido, a visão contextualizada do indivíduo e de seus problemas atuais ou potenciais de saúde é bastante enfatizada. Além disso, chama-se a atenção para a necessidade de se desenvolver pesquisas sobre o papel do enfermeiro na comunidade e no hospital, a partir da visão da antropologia social (Noble, 1964).

$\mathrm{Na}$ Espanha, devido às particularidades do seu sistema de ensino superior, os cursos de Enfermagem são oferecidos apenas no nível de primeiro ciclo ou diplomatura, o que não habilita o profissional ao ingresso em cursos de pós-graduação strictu sensu (mestrado e doutorado). Nesse sentido, muitos enfermeiros têm cursado a licenciatura em Antropologia como forma de completar o nível de segundo ciclo e poderem ingressar em mestrados e doutorados (terceiro ciclo). Esta particularidade tem favorecido $o$ fortalecimento da relação entre enfermagem e antropologia na Espanha, o que pode ser evidenciado pela constituição de um subcampo específico: a Antropologia dos Cuidados (González Gil, Martínez Gimeno \& Luengo González, 2006).

A Antropologia dos Cuidados guarda as influências da obra de Madeleine Leininger e da Antropologia Cultural norte-americana. No âmbito nacional, destacam-se as figuras dos professores Manuel Amezcua e José Siles González. O país conta com as revistas

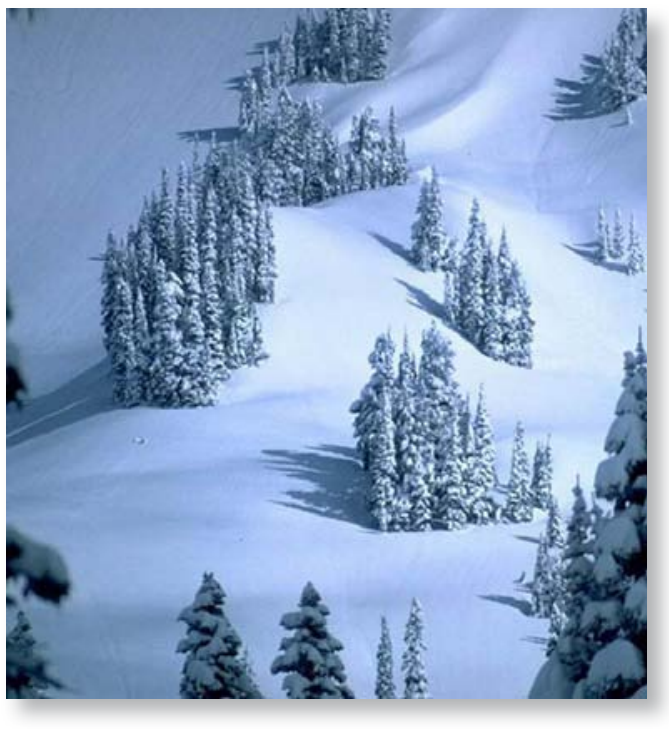

"Index de Enfermería" criada em 1988 e com a revista "Cultura de los Cuidados: Revista de Enfermería y Humanidades" especializada em Antropologia dos Cuidados e publicada pela Universidade de Alicante, desde 1997. A Antropologia dos Cuidados tem ampla penetração nos países ibero-americanos, notadamente nos de língua espanhola da América Latina e, pouca circulação em países de outras línguas, principalmente a inglesa.

$\mathrm{O}$ foco de atenção da Antropologia dos Cuidados é o estudo tanto do setor especificamente profissional, como dos cuidados informais/domésticos, dos cuidados derivados de interpretações mágicas, religiosas ou, simplesmente, fundamentadas nos usos e costumes de uso repetido através do tempo em um mesmo lugar até adquirir o caráter de tradição normativa ou prescritiva. Os autores defendem, também, o paradigma sociocrítico como base epistemológica da Antropologia dos Cuidados, com destaque especial à obra de Jürgen Habermas (2002).

Nesse sentido, a Antropologia dos Cuidados possui três especialidades: 1) a Enfermagem Transcultural que enfatiza o 
estudo comparativo e sistemático intercultural para identificar as diferenças culturais que dão lugar às distintas formas de praticar e sentir o cuidado; 2) a Antropologia Clínica, mas preocupada com os contextos de prática profissional oficiais; e 3) a Antropologia Educativa dos Cuidados, relacionada aos estudos sobre educação em saúde (Siles González \& Solano Ruiz, 2009).

Cumpre salientar que, assim como nos EUA, a enfermagem espanhola tem uma importante coesão sociocorporativa, o que tem fortalecido a Antropologia dos Cuidados e suas influências em vários países de língua hispânica através das atividades da Asociación de Historia y Antropología de los Cuidados, com sede em Alicante. Esta associação promove jornadas e congressos anuais e bienais, e tem se constituído como um importante fórum de discussão sobre temas relacionados à história, à fenomenologia e à antropologia em suas relações com a enfermagem.

Conforme se observa, o desenvolvimento da relação entre enfermagem e antropologia nesses países em muito reflete os estímulos que moveram os primeiros antropólogos na condução dos seus estudos: o interesse pelo Outro. Tanto nos Estados Unidos, como na Espanha e Inglaterra, as mudanças no perfil demográfico ocasionadas, principalmente, pela migração, confluíram para a existência de uma diversidade cultural na população em geral. Em última instância e, sobretudo, recentemente, temas como transculturalismo, multiculturalismo e migração têm ocupado importantes espaços na agenda política, econômica e social desses países. Nesse contexto, a tradição culturalista norteamericana tem-se mostrado uma ferramenta teórica e metodológica acessível e de fácil operacionalização pelos enfermeiros no intuito de compreenderem esse pluralismo humano e orientar suas práticas.

Além disso, a própria história e organização da disciplina e profissão de enfermagem nesses países, tanto no nível de formação acadêmica como na inserção no mercado de trabalho, se reflete na produção de uma identidade profissional aparentemente mais bem delineada, com maior clareza do foco de atuação e maior domínio e utilização dos referenciais teóricos da enfermagem. Penso que esses aspectos influenciem na postura do enfermeiro nas suas inserções acadêmicas e na prática assistencial. No que tange ao campo acadêmico, por hora a melhor expressão que se poderia utilizar seria uma postura “empreendedora", no sentido de conseguirem visualizar lacunas e propor avanços condizentes com a realidade, pensamento estratégico, visão de futuro, criatividade, articulação política dentro e fora do país, para citar algumas características.

$\mathrm{O}$ que quero salientar é o pioneirismo e a tendência ao desenvolvimento de um pensamento que, mesmo mantendo suas ligações com as correntes teóricas e suas tradições, propõe avanços na pesquisa, no ensino e na prática assistencial por meio de criações de novos modelos teóricos, novos instrumentos de avaliação clínica e novas metodologias e estratégias de ensino, por exemplo. Nesse sentido, pretendo contrastar com o caráter reprodutivo que ainda se insinua, principalmente, nas pesquisas em enfermagem no Brasil. Talvez essa condição reflita uma interpretação às avessas do estímulo à internacionalização tão presente no discurso dos órgãos governamentais brasileiros, o que, no meu entender, manifestase por meio de um mimetismo das tendências internacionais sem, contudo, realizar as 
devidas "traduções" e adaptações para o nosso contexto e a valorização das potencialidades do pensamento e da produção científica nacional.

\section{Relações e Dilemas entre Enfermagem e Antropologia no Brasil}

No Brasil, o contexto social, político e educacional no qual se inicia a aproximação e relação entre Enfermagem e Antropologia foram os anos 1980. Uma década marcada pelo movimento de redemocratização do país e pela organização da sociedade civil. $\mathrm{Na}$ área de saúde, especificamente, havia o Movimento da Reforma Sanitária com ressonâncias tanto na formação quanto no trabalho em saúde como um todo. $\mathrm{Na}$ enfermagem, os programas de pós-graduação strictu-sensu passavam por um importante processo de consolidação, caracterizado, sobretudo, pela criação do primeiro Programa de Doutorado em Enfermagem da América Latina, na Universidade de São Paulo (USP). Nesse caldo político e cultural, as enfermeiras brasileiras, principalmente as docentes das escolas e faculdades de enfermagem, iniciaram uma reflexão para compreender a crise da enfermagem brasileira e sua inserção no campo do trabalho em saúde. É nesse período que se busca a realização de pesquisas qualitativas em enfermagem, tendo como referencial teórico-metodológico o materismo histórico-dialético.

O marco da aproximação entre enfermagem e antropologia foi a vinda da Dra Madeleine Leininger por ocasião do "Io Encontro Interamericano de Pesquisa Qualitativa em Enfermagem", realizado em 1988 na cidade de São Paulo, por meio da parceria entre a Escola de Enfermagem da USP e o Departamento de Enfermagem da Universidade Federal de Santa Catarina (UFSC). As idealizadoras desse evento foram a Dra Ingrid Elsen (UFSC) e a Dr ${ }^{\text {a }}$ Maguida Stefanelli (USP).

Leininger, em seu pioneirismo, tinha uma visão sobre a enfermagem, a antropologia e o cuidado cultural - conceito central de sua teoria - que veio de encontro às necessidades das enfermeiras brasileiras da época. O evento possibilitou a ida de doutorandas e docentes aos EUA para aprender o processo de pesquisa qualitativa e as bases da Enfermagem Transcultural. De volta ao país, elas continuaram a usar o método com alunos e colaboradores, disseminando assim o conhecimento adquirido e abrindo espaço para a pesquisa qualitativa na enfermagem com forte inspiração na Teoria da Diversidade e Universalidade do Cuidado Cultural, publicada anos depois por Leininger no livro Culture Care Diversity and Universality: a theory of nursing (Leininger, 1991).

Paralelamente, nos programas de pósgraduação em Antropologia Social e em Saúde Pública/Saúde Coletiva, desenvolviamse os primórdios da Antropologia da Saúde brasileira. Nesses campos era comum a influência de pesquisadores formados em universidades estrangeiras, a exemplo de Marcos de Souza Queiroz e de Maria Andrea Loyola, com doutorados cursados na Inglaterra e França, respectivamente (Langdon \& Follér, 2012). Na enfermagem, esse mesmo processo ocorre por meio da Dra Ingrid Elsen cujo doutorado foi feito nos EUA.

A influência da teoria de Leininger nas pesquisas brasileiras foi maior entre a segunda metade da década de 1990 e a primeira metade dos anos 2000. Contrariamente aos enfermeiros norte-americanos e espanhóis, os brasileiros não se organizaram em associações, comitês, sociedades ou conselhos para o fortalecimento dos estudos antropológicos 
ou transculturais em Enfermagem. Podemos ainda questionar o porquê de não termos relações nem representatividade dentro da Transcultural Nursing Society. Esta é uma indagação inquietante, uma vez que esta Sociedade tem como objetivo consolidar e disseminar a Enfermagem Transcultural em todo o mundo e os convênios entre universidades e institutos de pesquisa são estimulados.

Ao mesmo tempo, dada a dificuldade de introduzir cursos de Enfermagem Transcultural nos cursos de graduação e pós-graduação, à obra de Leininger passou a circular em espaços isolados e principalmente entre pesquisadores que desenvolviam estudos transculturais. Pode-se cogitar que um fator impeditivo à consolidação da Enfermagem Transcultural no Brasil tenha sido o enfoque dado por Leininger nas minorias étnicas, imigrantes, estrangeiros. Nos anos 1990, por exemplo, na população brasileira não havia número significativo de imigrante ou estrangeiro. Por outro lado, o debate sobre transculturalismo era incipiente, como ainda o é, valorizando-se a cultura brasileira como uma unidade apesar das diferenças internas e regionalismos.

Portanto, o debate transcultural marcado pelos enfoques em imigrantes não fazia sentido no contexto brasileiro de então. Ainda assim, produziram-se alguns estudos realizados por pesquisadores da Universidade Federal de Santa Catarina, da Universidade Federal do Rio Grande do Sul e da Universidade Federal do Paraná. Outras universidades (Universidade de São Paulo e Universidade Federal do Ceará, principalmente) também contam com pesquisas realizadas com este referencial teórico-metodológico (Oriá, Ximenes \& Alves, 2006). Mas, esse tipo de pesquisa tem diminuído e, o mais intrigante, seus resultados e reflexões têm tido pouco impacto na prática assistencial da enfermagem nos variados tipos de serviços de saúde.

Na verdade, as grandes influências da $\mathrm{Dr}^{\mathrm{a}}$ Leininger no Brasil foram: i) disseminação da pesquisa qualitativa em enfermagem; e ii) posteriormente, aproximação das enfermeiras com o campo da Antropologia. Porém, é justamente nesse contato com a Antropologia que as enfermeiras começam a trilhar um caminho próprio e concatenado com o desenvolvimento das Ciências Sociais e Saúde no Brasil.

Os cursos oferecidos na pós-graduação sobre teorias e métodos da antropologia e outras ciências sociais pôs os pesquisadores da Enfermagem em contato com o campo da Antropologia da Saúde brasileira que vinha se consolidando desde os anos 1970. Da relação com antropólogos especializados em saúde, tanto nos cursos quanto em bancas examinadoras de dissertações e teses, as enfermeiras, gradativamente, passaram a se interessar por outros referenciais teóricos que vinham sendo trabalhados por aqueles pesquisadores. Nesse sentido, nos últimos sete anos pode-se perceber a influência da Antropologia Cultural norte-americana (principalmente as obras de Byron Good, Arthur Kleinman e Clifford Geertz), do Interacionismo Simbólico e a permanência da Teoria do Cuidado Cultural de Leininger, em menor escala.

Esse "caminho" que vem sendo construído pelos enfermeiros brasileiros nas suas relações com a antropologia ou, mais especificamente com a antropologia da saúde ou médica, apresenta, todavia, alguns "riscos" ou "descaminhos". Se pararmos para analisar a produção brasileira de estudos 
culturais ou antropológicos na enfermagem, provavelmente nos depararemos com dois grupos de pesquisas: 1) estudos que tomam como objetos o processo de cuidar em enfermagem nas diversas fases do ciclo vital e em diferentes cenários de prática, bem como o processo de trabalho em enfermagem; e 2) os estudos que abordam aspectos mais gerais do processo saúde-doença sem uma vinculação direta com o processo de cuidar em enfermagem, as instituições de cura, seus práticos e o processo de trabalho em saúde.

No primeiro grupo de estudos há um delineamento parcial de uma Anthropology of nursing (Antropologia da enfermagem), uma vez que trabalham com as características socioculturais que definem a profissão: suas práticas, tradições e rituais. O segundo grupo desenvolve uma Anthropology in or for nursing (Antropologia em ou para enfermagem) já que estão mais preocupados com as formas pelas quais os conceitos e teorias antropológicas podem ser aplicados à prática da enfermagem (Holden \& Littlewood, 1991). Ainda como uma especificidade brasileira, o segundo grupo é mais influenciado pela antropologia da saúde geralmente por serem orientados por antropólogos especializados em saúde e credenciados nos Programas de Pós-graduação em Enfermagem. Enquanto o primeiro grupo é orientado, principalmente, por enfermeiros mestres e/ou doutores em Antropologia ou Ciências Sociais ou que desenvolveram sua formação e suas pesquisas com referenciais antropológicos mesmo vinculados a Programas de Pós-graduação em Enfermagem.

Feita essa "classificação", cabe salientar as potencialidades dessas investigações e o quanto esse tipo de conhecimento tem se revertido em práticas de cuidado profissionais mais congruentes com a realidade sociocultural dos usuários dos serviços de saúde e em uma perspectiva crítica das condições e da maneira como o processo de trabalho é organizado e executado. Por outro lado, cumpre igualmente destacar que esses grupos não têm promovido um debate sobre as relações da enfermagem com a antropologia no ensino, na pesquisa e na prática assistencial. Ou seja, embora haja um número significativo de estudos empíricos, seus resultados têm trazido poucos avanços epistemológicos, uma vez que, em geral, nem fazemos uma Antropologia da enfermagem nem uma Antropologia em ou para enfermagem.

Nessa perspectiva, o "descaminho" que a enfermagem brasileira tem trilhado na sua relação com a antropologia consiste no distanciamento das questões próprias da prática, do ensino e da pesquisa em enfermagem e na aproximação dos temas mais ligados à doença, seus sintomas, tratamentos e os sistemas médicos - preocupações da Antropologia da Saúde ou Médica.

Diferentemente do movimento ocorrido em países como Estados Unidos, Inglaterra e Espanha que desenvolveram suas relações com a antropologia de forma a atender as necessidades da enfermagem, com institucionalização e permeabilidade no meio acadêmico e assistencial, no Brasil, vê-se uma vinculação à Antropologia da Saúde. O que há de problemático nessa vinculação é a invisibilidade da produção acadêmica dentro da própria enfermagem e, principalmente na Saúde Coletiva e nas Ciências Sociais.

Portanto, pode-se afirmar que as relações entre enfermagem e antropologia no Brasil: nem se aproximaram de tradições já existentes em países como Estados Unidos (Enfermagem Transcultural) e Espanha (Antropologia dos 


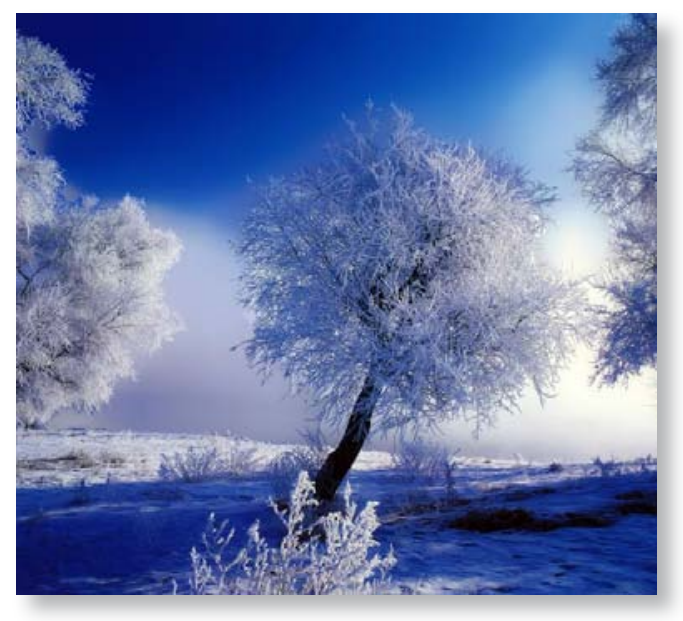

Cuidados); nem desenvolveu uma tradição teórico-metodológica própria ou que, de alguma maneira, se relacionasse com as já existentes fora do país; nem se inseriu nas agendas de pesquisas propriamente antropológicas ligadas, sobretudo, aos departamentos de Saúde Coletiva e Antropologia.

Dessa forma, alguns questionamentos precisam ser feitos no intuito de instigar reflexões e práticas. Estes dilemas na relação entre enfermagem e antropologia no Brasil:

1) Seria algo relacionado à subjetividade produzida no enfermeiro em sua formação profissional?

2) Seria devido à "crise" de identidade profissional do enfermeiro?

3) Seria devido ao "fetiche" do brasileiro pelo estrangeiro que, em alguma medida, acentua as consequências do processo de colonização que também impacta o desenvolvimento cultural e científico do país?

4) Seria despreparo do enfermeiro para uma visão empreendedora na profissão, principalmente no tocante ao estabelecimento de relações internacionais que promovam verdadeiros intercâmbios e não apenas um movimento unidirecional do que vem de fora do país para dentro dele?
5) Seria falta de ousadia, criatividade intelectual e organização política do enfermeiro?

6) Seria a dificuldade em desenvolver um pensamento crítico e reflexivo independente de outros profissionais, mas sem perder de vista a interdisciplinaridade?

7) A aproximação com a Transcultural Nursing Society e com a Asociación de Historia y Antropología de los Cuidados seria uma forma eficaz e estratégica de fortalecer esse subcampo no Brasil?

\section{Desafios ou encaminhamentos}

O objetivo deste ensaio foi promover uma breve discussão sobre enfermagem e antropologia pontuando as particularidades do contexto brasileiro em comparação com Estados Unidos, Inglaterra e Espanha e, principalmente, problematizar os dilemas (problemas e limites) existentes nas relações entre as duas disciplinas e indicar alguns desafios para seu desenvolvimento e consolidação. Nesse sentido, o arrolamento dos argumentos e a análise comparativa da construção dessa relação em diferentes países permitiram visualizar algumas hipóteses que visam contribuir para estudos e discussões futuras.

Como desafios podem ser apontados: a premência da constituição do subcampo da Antropologia da Enfermagem no Brasil, bem como seu delineamento epistemológico; a sistematização da produção acadêmica já existente no tocante à relação entre enfermagem e antropologia com vistas a identificar as potencialidades, limites e lacunas desse conhecimento; a proposição de modelos teóricos ou teorias de enfermagem com essa perspectiva teórica e metodológica e suas interfaces com o processo de enfermagem, 
de maneira que se possam produzir impactos significativos na prática de enfermagem em diversos cenários de atuação profissional; a representação política dos pesquisadores e estudantes desse subcampo por meio da organização de associações e redes (networks); e a introdução de disciplinas específicas com abordagem sociocultural do cuidado, da saúde e da doença, bem como dos processos de trabalho em enfermagem, nos cursos de graduação (além das disciplinas básicas de Sociologia e Antropologia), por exemplo, Enfermagem Transcultural ou Antropologia dos Cuidados (ou com outras denominações), ministradas por enfermeiros com formação e/ou experiências assistenciais e de pesquisas nesse subcampo profissional.

\section{REFERÊNCIAS}

- Cartana, M.H.F. \& Heck, R.M. (1997) Contribuições da antropologia na enfermagem: refletindo sobre a doença. Texto \& Contexto Enfermagem, 6 (3), 233-40.

- Dougherty, M.C. \& Tripp-Reimer, T. (1985) The interface of nursing and anthropology. Annual Review of Anthropology, 14, 219-41.

- González Gil, T; Martínez Gimeno, L; Luengo González, R. (2006) Antropología de los cuidados en el ámbito académico de la enfermería en España. Texto \& Contexto Enfermagem, 15 (1), 155-61.

- Habermas, J. (2002) Teoría y praxis. Tecnos, Barcelona.

- Holden, P. \& Littlewood, J. (1991) Anthropology and nursing. Routledge. London.

- Langdon, E.J. \& Follér, M. (2012) Anthropology of health in Brazil: a border discourse. Medical Anthropology, 31 (2), 4-28.

- Leininger, M.M.(1970) Nursing and anthropology: two worlds to blend. Wiley. New York.

- Leininger, M.M. (1991) Culture care diversity and universality: a theory of nursing. National League for Nursing. New York.
- Leininger, M.M. (2001) Current issues in using anthropology in nursing education and services. Western Journal of Nursing Research, 23 (8), 795-806.

- Littlewood, J. (2001) A model for nursing using anthropological literature. International Journal of Nursing Studies, 26 (3), 221-9.

- Martorell Poveda, M.A. (2001) ¿Antropoenfermería o enfermeantropología? Cultura de los Cuidados, 5 (9), 5-9.

- Mulhall, A. (1996) Anthropology, nursing and midwifery: a natural alliance? International Journal of Nursing Studies, 33 (6), 629-37.

- Noble, M. (1964) Social anthropology and nursing. International Journal of Nursing Studies, 1, 159-63.

- Oriá M.O.B; Ximenes, L.B; Alves, M.D.S. (2006) Utilização da Teoria do Cuidado Cultural na pósgraduação em enfermagem: a realidade brasileira. Revista de Enfemagem UERJ, 14 (2), 245-52.

- Queiroz, M.S. (2003) Saúde e doença: um enfoque antropológico. Bauru: Edusc.

- Siles González, J; Cibanal Juan, L; Vizcaya Moreno, M.F; Gabaldón Bravo, E.Ma; Domínguez Santamaría, J.M; Solano Ruiz, Ma .C., et al. (2001) Una mirada a la situación científica de dos especialidades esenciales de la enfermería contemporánea: la antropología de los cuidados y la enfermería transcultural. Cultura de los Cuidados, 5 (10), 72-87.

- Siles González, J. y Solano Ruiz M.C. (2009) Clarificación epistemológica de tres especialidades de la antropología de los cuidados: antropología clínica, enfermería transcultural y antropología educativa de los cuidados. En: Siles González, J. y Solano Ruiz, M.C. (coordinadores). Antropología educativa de los cuidados: una etnografía del aula y las prácticas clínicas. Universidad de Alicante; Editorial Marfil, Alicante:11-66. 